\title{
Electrical injuries in adult patients - 3 years overview
}

\author{
Andreea GROSU-BULARDA ${ }^{1}$, Mihaela-Cristina ANDREI ${ }^{1,2}$, Adrian FRUNZA ${ }^{1,2}$, \\ Florin-Vlad HODEA ${ }^{1}$, Sabina GRAMA ${ }^{1}$, Ioan $\operatorname{LASCAR}^{1,2}$, Razvan Nicolae TEODOREANU ${ }^{1,2}$ \\ ${ }^{1}$ Clinic of Plastic Surgery, Aesthetic and Reconstructive Microsurgery, Clinical Emergency Hospital, \\ Bucharest, Romania \\ 2 "Carol Davila" University of Medicine and Pharmacy, Bucharest, Romania
}

\begin{abstract}
Electrocutions are a particular type of trauma, usually affecting young active people, leading to high morbidity and mortality rates in extensive injured patients. Those patients require complex, multidisciplinary treatment in specialized burn centers.

We conducted a three-year retrospective study in the Burn Unit of the Clinical Emergency Hospital Bucharest, Romania, aiming to identify different factors that characterize electrical injuries, with the goal to improve our clinical practice, in order to decrease overall complications, the morbidity and mortality rates and obtain an optimal functional prognosis for those severely injured patients. Patient-related and injury-related parameters were analyzed, and particularities observed in our burn unit were noted.

A clear understanding of the physiopathology of those injuries and their complications is essential for providing an optimal therapeutic strategy. Rapid initiation of systemic supportive measures, accurate diagnostic and an adequate surgical treatment, correctly conducted, are essential for improving the vital and functional prognostic of patients who suffer electric injuries.
\end{abstract}

Keywords: electrical injuries, severity factors, surgical treatment, fasciotomies, complications

\section{INTRODUCTION}

Electrocutions are a particular type of trauma, divided clinically into two types: high voltage lesions and low voltage lesions. The threshold between the two types of lesions is $1000 \mathrm{~V}$. Low voltage electrical injuries are similar to thermic burns and usually present full dermal thickness and subdermal cutaneous lesions $[1,2]$.

High voltage electrical injuries may determine various degrees of cutaneous burns, associated with hidden and ample profound cutaneous lesions, also called "iceberg tip" lesion. This type of injury determines progressive tissular necrosis, which surpasses the ampli- tude and localization of cutaneous lesions. The lesions are similar to crush-type traumas, due to both thermal injuries, as well as micro- and macrovascular local changes. Although the lesion is localized, the metabolic immediate effect is directly proportionate to the affected tissues quantity and initial vascularization status of respective tissues [1-5].

Electrical injuries can be divided by type into four categories: true electrical injury, lightning injury, flame, and flash. The latter, usually results in superficial burns, due to an arc flash, the patient suffering no electrical injury, with no current passing through the skin. Flame injuries may determine clothing ignition, in addition to passage or not of electrical current. As far as lightning 
injuries are concerned, high voltage electrical current passes throughout the patient's body in a short period of time. Lastly, patients become part of the current circuit when suffering a true electrical injury, hence the presence of entry and exit wounds $[2,6]$.

For high voltage injuries, the lesions can be fatal instantly. Nevertheless, the damage associated with electrocutions can result in multiple tissues or organs dysfunction, affecting different types of tissues both superficial and profound [2]. Table 1 illustrates specific injuries that may occur in electrocutions [3].

TABLE 1. Tissues affected by electrical burns [3]

\begin{tabular}{|c|c|}
\hline $\begin{array}{l}\text { Damaged } \\
\text { tissue }\end{array}$ & Injury type \\
\hline Skin & Thermal injury \\
\hline $\begin{array}{l}\text { Muscular } \\
\text { system }\end{array}$ & $\begin{array}{l}\text { Necrosis } \\
\text { Compartment syndrome }\end{array}$ \\
\hline Osteo-skeletal & $\begin{array}{l}\text { Fractures } \\
\text { Sprains } \\
\text { Joint dislocation }\end{array}$ \\
\hline Cardio-vascular & $\begin{array}{l}\text { Vascular necrosis } \\
\text { Vascular thrombosis } \\
\text { ST elevation (most common EKG change) } \\
\text { Dysrhythmias } \\
\text { - Supraventricular dysrhythmias } \\
\quad \text { Atrial fibrillation (most common } \\
\text { dysrhythmia) } \\
\text { Sinus tachycardia } \\
\text { Atrial extrasystoles } \\
\text { - Ventricular fibrillation (most common } \\
\text { cause of fatality) } \\
\text { Bradycardia } \\
\text { Cardiac ischemia and myocardial infarction }\end{array}$ \\
\hline GI tract & Intestinal necrosis \\
\hline Renal system & $\begin{array}{l}\text { Myoglobinuria } \\
\text { Acute tubular necrosis } \\
\text { Acute renal failure following rhabdomyolysis }\end{array}$ \\
\hline Ocular system & Cataract \\
\hline
\end{tabular}

The prompt surgical treatment, correctly conducted, is essential for improving the vital and functional prognostic of patients who suffer electric injuries [3].

\section{MATERIAL AND METHODS}

We conducted a three-year retrospective cross-sectional study in the Burn Unit of the Clinical Emergency Hospital, Bucharest, Romania, between May 2016 and May 2019, where a single lot of 23 patients was gathered. Criteria for study admission were based up mechanism of action, with inclusion of patients who suffered electric burns either high or low voltage electric injuries, excluding patients with scald, contact, fire, chemical or radiation burns. Multiple parameters were observed for each patient: demographic characteristics, lesion characteristics, encountered complications and patient outcome. In order to gather the data, we used the Burn Unit's both digital archives (eHealth program, HIPOCRATE) and physical archives. Formal consent was obtained from all patients belonging to this study. Analysis of data was performed using statistics software: Microsoft Excel and IBM SPSS.

\section{RESULTS}

23 electrocutions occurred during a three-year period in our unit. All of them were men. Total burn patients were 269 , meaning $8.5 \%$ were electrocutions. 10 (43\%) patients who suffered electrical injuries died and $2(9 \%)$ of them were transferred to other burn units in other countries. Almost half of the patients survived (48\%). Regarding home environment, 14 (61\%) patients presented urban origins, the rest of $9(39 \%)$ having rural origins.

We observed the modality of hospital admission: 13 (57\%) patients were referred to our burn care unit from other hospitals, while $10(43 \%)$ patients presented directly to our emergency department. Notably, 21 out of 23 patients $(91.3 \%)$ patients belong to the active age population, falling within the working age range, while the other two were elderly patients ( $>80$ years of age) (Fig. 1).

Total body surface area (TBSA) burns percentage is evenly distributed amongst all categories in patients who suffered electric burns, notably, the most represented surface range was $61-70 \%$ (Fig. 2).

Most of the patients, 20 out of $23(87 \%)$, presented with $3^{\text {rd }}$ degree burns after electrocution. Only three (13\%) patients suffered from more superficial skin lesions. In addition, almost one third of the patients presented an airway injury, another negative prognostic factor on the patient's outcome. All anatomical segments were affected in electrocutions, with emphasis on the upper body area consisting of cephalic extremity, thorax and upper limbs (a combined $61 \%$ of all body) (Fig. 3).

Survival predictability was reliable, achieved through usage of the ABSI (abbreviated burn severity index) score (Fig. 4).

There were 11 flash-type etiological electrical injuries, out of which 3 were true electrocution combined with flash injuries, all of them being included in the high voltage category. Out of the 8 flame-type injury there was 1 injury consisting of both true electrocution and flame made, also included in the high voltage category. None of the patients suffered a lightning injury (Fig. 5).

Almost half (12 cases) of the electrical burns occurred in a workplace set environment. Two thirds of the patients admitted with electrical burns required mechanical ventilation. The minim number of mechanical ventilation hours was 7 hours, while the maximum 


$$
\begin{array}{r}
14 \\
12 \\
10 \\
8 \\
6
\end{array}
$$

\section{2}

8

4

2

0

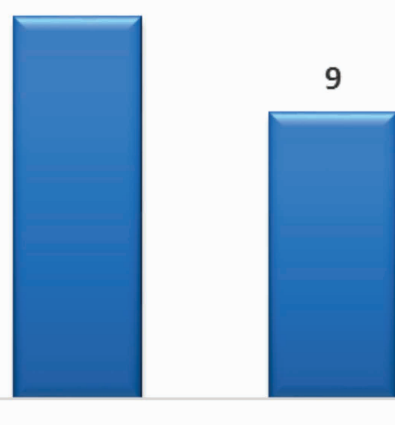

0

2

18-40 years

41-60 years

FIGURE 1. Patients distribution by age groups

5

4

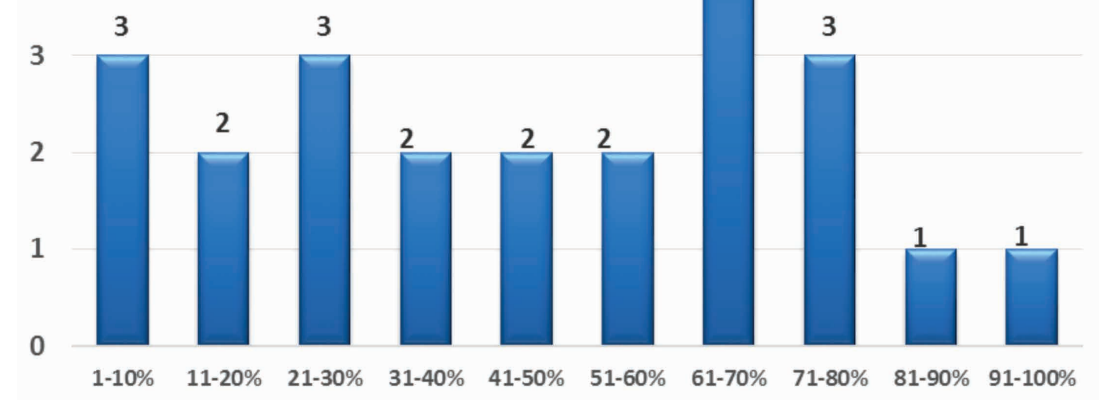

FIGURE 2. TBSA distribution

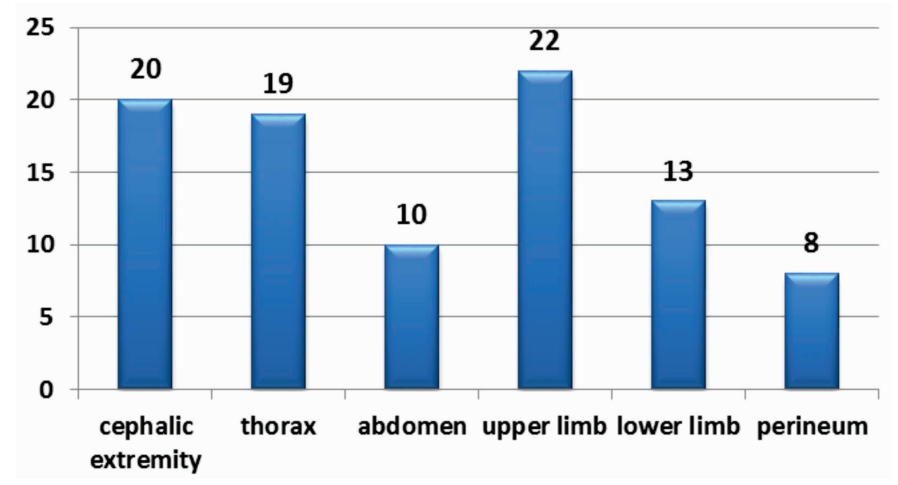

FIGURE 3. Burn areas affected by electrical injuries

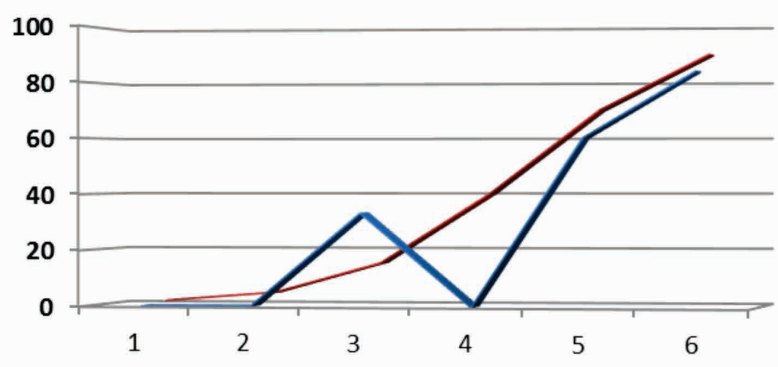

n Observed mortality

n expected mortality

FIGURE 4. Correlation between the ABSI score and mortality 


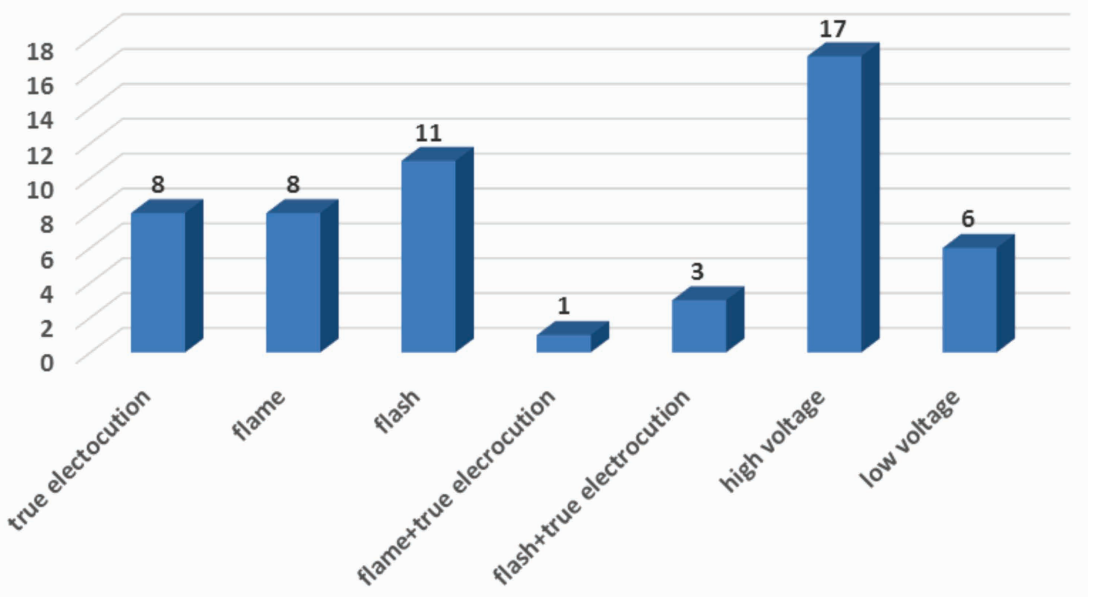

FIGURE 5. Type of electric injury

was 7,200 hours. The average number of mechanical ventilation hours were 427 , with a standard deviation of 1,452 , which indicated non-clustered data results with a widespread distribution.

Mean hospitalization days were 34 days, with a standard deviation of 60 . The shortest admitted period was 1 day and the largest was 303 days. More than a third (39\%) of all patients developed a complication. More than half of them (55\%) presented infectious complications, followed by neurological complications, cardiovascular, renal and gastrointestinal. Pneumonia was the most common finding amongst the infectious complications, followed by urinary tract infections, wound infection and gastrointestinal tract infection. One patient developed both infectious and neurological complications, while another both infectious and gastrointestinal complication (Fig. 6).

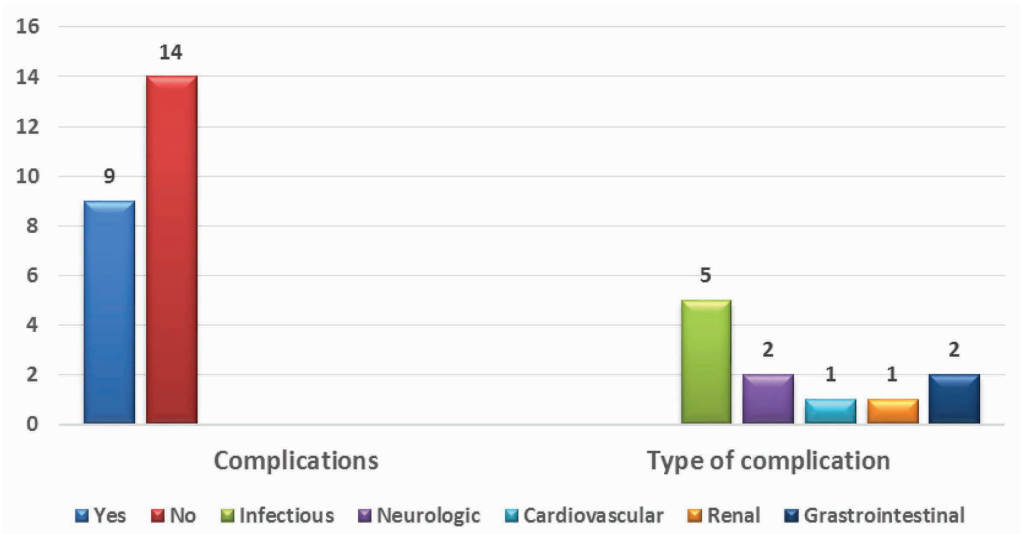

FIGURE 6. Electrical burns complications

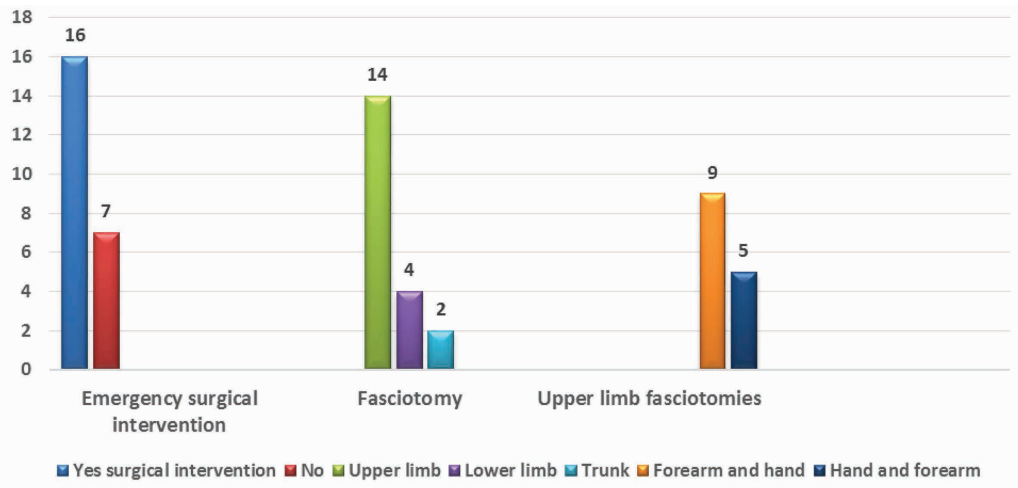

FIGURE 7. Emergency surgical interventions 
Only 3 (13\%) patients presented with comorbidities, one patient presented elevated blood pressure, another patient presented type II diabetes and elevated blood pressure, while the latter presented elevated blood pressure, neoplasia and stable angina pectoralis.

Two thirds of the patients required emergency surgical intervention, 14 (60\%) needing immediate fasciotomy, $2(9 \%)$ needing amputations. Out of all fasciotomies, 14 were performed in the upper limb, while 4 were performed in the lower limb and 2 in the trunk and abdomen (Fig. 7).

\section{DISCUSSION}

Electrical injuries are uncommon but are recognize as the most devastating type of burn injuries, with high morbidity and mortality, determining severe damage of both the skin and deep tissues, leading to large necrosis areas. Those patients require complex, multidisciplinary treatment in specialized burn units $[7,8]$.

Through this study we tried to identify different factors that characterize electrical injuries, with the goal to standardize and improve our clinical protocols in order to decrease overall complications, the morbidity and mortality rates and obtain an optimal functional prognosis for those severely injured patients. Patient-related and injury-related parameters were analyzed and particularities observed in our burn unit were noted.

National Burn Repository (NBR) of the American Burn Association (ABA) reports for 2016 stated that 486,000 patients presented burn injuries requiring medical treatment, 40,000 required hospitalizations with a survival rate of $96.8 \%$. Electrical injuries represented the admission cause for $4 \%$ of those patients [9].

In our study group of 269 patients admitted in our Burn Unit on the three years period, we observed that 8.5 of the cases suffered electrical injuries, an incidence two-fold higher than data usually reported by other centers.

All our patients were males, $91.3 \%$ of them belonging to the working active age population, half of the electrical injuries occurring in a workplace set environment. Electrical injuries occur in both young and labor active male individuals, as resulted from our study. Those findings are concordant with available literature data [7].

More than half of the patients were admitted in our burn unit after being transferred from other hospitals, sometimes requiring long-distance transport. This aspect may lead to inappropriate emergency treatment, delays fluid resuscitation and adequate surgical treatment, posing also transport related-risk and higher infectious risk.
Regarding the etiology, three quarters of cases presented high voltage electric injuries.

In this study, electrical injuries associated also extensive burns, with two thirds of the patients having over $30 \%$ TBSA burns, attesting a poor prognostic. 13 of 23 patients presented burn injuries over 40\% TBSA. Morbidity and mortality risk increased with TBSA in a linear manner, burns over 40\% TBSA in adult patients determining high risk for severe complications and higher mortality rate, even in the setting of high-quality standard of care [10].

Most of the patients, 20 out of 23 (87\%), presented with full thickness burns after electrocution. Only three (13\%) patients suffered more superficial burn lesions. Presence of third-degree burn is an important severity factor. Correct surgical treatment with early excision and grafting of the full thickness burn wounds is mandatory to reduce risk of complications and improve patient outcome [11].

We emphasize the importance of surgical treatment in management of electrical injuries cases, in order to reduce morbidity and mortality and promote and adequate recovery of those patients.

Emergency protocols contains the following maneuvers. Firstly, excision of constituted areas of necrosis from both entry and exit wounds is performed, as well as skin ischemic areas that appeared after passage of electrical current or thermic burns, following electrical arch or burning clothes. After thorough excision, the next steps are either: Decompression incisions at the level of necrosed areas and third-degree burns, as well as at the level of the limbs where a compartment syndrome is suspected, either fasciotomies and fasciectomies of the limbs, usually at the level of the forearms, hands and calves, excision of devitalized muscles, or necessity amputations in case of extremity carbonization or in case of constituted ischemia.

Post-emergency surgical treatment includes daily dressing of the wounds, excision of septic tissues post-necrosis or post-deepening of the burns, excision of the areas where ischemia aggravated or where necrosis developed, even necessity amputations in case of major arterial source lesion, without possible of coverage with quality biological materials. Lastly, reconstructive surgery is performed to cover post-excision defect through either skin grafts, local or distant flaps.

In this study, $69 \%$ patients required emergency surgical intervention, 14 (60\%) needing immediate fasciotomy, 2 (9\%) needing amputations. For 14 patients, fasciotomies were performed in the upper limb, while 4 were performed in the lower limb and 2 in the trunk and abdomen. More often, fasciotomies of the hand and forearm were performed than arm and forearm.

In our patients, we observed extensive involvement of functional areas, leading to more demanding surgi- 
cal strategies and necessity for long term patient follow up to improve functional prognostic of the patients.

Presence of inhalation injuries in burn trauma is correlated with high morbidity and mortality rates. The inhalation injury is evaluated using fiber-optic bronchoscopy, being reported a direct correlation between the inhalation injury severity observed at bronchoscopy and patient's mortality [12-14]. One third of our patients with electrical injuries also associated inhalation injuries, leading to poor prognosis for those cases.

Two thirds of the patients admitted in our unit with electrical burns required mechanical ventilation. The minim number of mechanical ventilation hours was 7 hours, while the maximum was 7,200 hours. The average number of mechanical ventilation hours were 427. A prolonged duration of mechanical ventilation determines a poor prognosis for the patient, causing multiple complications. Patients with extensive burns needing mechanical ventilation for long term and requiring multiple surgical procedures are candidates for tracheostomy procedure $[15,16]$.

We observed very high mortality levels in our group, therefore we analyzed the correlation between observed mortality and mortality predicted trough ABSI (abbreviated burn severity index) score correlates with expected mortality in our patients. ABSI score is an important predictor of mortality in burned patient's population, maintaining its scientific value for almost four decades, since it was introduced by Tobiasen in 1982 $[17,18]$. We must take into consideration each severity factor encountered in the patients to adjust the therapeutic strategy.

It is well known that electrical injuries associate severe systemic complications.

Cardiac arrest appears during either first-time presentation or at the end of a long and complicated admission case. Continuous cardiovascular monitorization is mandatory in electrocution cases due to high risk of cardiac complications [19].

Many of the complications are similar to those of the burns, crush syndromes, including infection, myositis, myoglobinuria. Relevant incidence of acute kidney failure due to myoglobinuria has decreased, given the aggressive liquid reanimation and adequate surgical treatment. Fasciotomies are necessary to prevent compartment syndromes and acute nerve compression. Devitalized tissue aggressive debridement and major amputations are usual needed in cases with severe, extensive tissue destruction, in order to prevent reperfusion complications and death $[3,4,20]$.

Neurological complications, usually high-voltage electrical injuries associated with such as loss of con- sciousness, peripheral or central nerve lesions and delayed lesions of the spinal cord were reported [21]. Long term complications neuropsychiatric complications include posttraumatic stress disorder, depression, anxiety, memory and attention impairment [22].

Stress ulcer, known as Curling ulcer, may developed in severely burned patients [23]. In electrocution cases, abdominal lesions due to ischemia, vascular lesions or blunt force trauma can be initially overlooked in.

Infectious complications are a common occurrence in burn unit's patients. The most frequent cause of hospital mortality is represented by pneumonia, sepsis, and multiple organ insufficiency [24].

In our group, we noticed that 9 of 23 all patients developed complications, more than half of them being infectious, followed by neurological complications, cardiovascular, renal and gastrointestinal. Pneumonia was the most common finding amongst the infectious complications, followed by urinary tract infections, wound infection, and gastrointestinal tract infection. One patient developed both infectious and neurological complications, while another both infectious and gastrointestinal complication.

The patient severely affected by electrical injuries represents a challenge for the plastic surgeon, besides live saving, the main desideratum being the fastest and most complete recovery of this type of patient. Unfortunately, not infrequently, due to the complexity of the lesions, significant sequelae persist and varying degrees of disability, requiring long term rehabilitation support for social reintegration.

\section{CONCLUSIONS}

Electrocutions are a particular type of trauma, usually affecting young active people, leading to high morbidity and mortality rates in extensive injured patients. Those patients require complex, multidisciplinary treatment in specialized burn units. Rapid initiation of systemic supportive measures, accurate diagnostic and an adequate surgical treatment, correctly conducted, are essential for improving the vital and functional prognostic of patients who suffer electric injuries. A clear understanding of the physiopathology of those injuries and their complications is essential for providing an optimal therapeutic strategy. It is not uncommon that electrical injuries victims to have poor functional recovery, requiring long term follow-up and integration in sustained rehabilitation programs.

Conflict of interest: none declared Financial support: none declared 


\section{REFERENCES}

1. Lee DH, Desai MJ, Gauger EM. Electrical Injuries of the Hand and Upper Extremity. J Am Acad Orthop Surg. 2019 Jan 1;27(1):e1-e8.

2. Zemaitis MR, Foris LA, Lopez RA, et al. Electrical Injuries. [Updated 2021 Jul 23]. In: StatPearls [Internet]. Treasure Island (FL): StatPearls Publishing; 2021.

3. Bernal E, Arnoldo BD. Electrical Injuries. In: Herndon DN (ed.). Total Burn Care (5th edition). Elsevier, 2018;396-402.e2.

4. Teodoreanu R, Popescu SA, Lascar I. Electrical injuries. Biological values measurements as a prediction factor of local evolution in electrocutions lesions. $J$ Med Life. 2014;7(2):226-236.

5. Manandhar K, Shrestha J, Rayamajhi S, Lohani I. Electrical Injuries: The Iceberg Phenomena. Journal of Society of Surgeons of Nepal. 2017;20(2):4-12.

6. Gentges J, Schieche C. Electrical injuries in the emergency department: an evidencebased review. Emerg Med Pract. 2018 Nov;20(11):1-20.

7. Kym D, Seo DK, Hur GY, Lee JW. Epidemiology of electrical injury: Differences between low- and high-voltage electrical injuries during a 7-year study period in South Korea. Scand J Surg. 2015 Jun; 104(2):108-14.

8. Jeschke MG, van Baar ME, Choudhry MA, Chung KK, Gibran NS, Logsetty S. Burn injury. Nat Rev Dis Primers. 2020;6(1):11.
9. American Burn Association (ABA)-Burn Incidence Fact Sheet: Burn Incidence and Treatment in the United States: 2016. Available at: https://ameriburn.org.

10. Jeschke MG, Pinto R, Kraft R, et al. Morbidity and survival probability in burn patients in modern burn care. Critical Care Medicine. 2015;43(4):808-815.

11. Rowan MP, Cancio LC, Elster EA, et al. Burn wound healing and treatment: review and advancements. Critical Care. 2015;19:243.

12. Dries DJ, Endorf FW. Inhalation injury: epidemiology, pathology, treatment strategies. Scandinavian Journal of Trauma, Resuscitation and Emergency Medicine. 2013;21:31.

13. Hassan Z, Wong JK, Bush J, Bayat A, Dunn $\mathrm{KW}$. Assessing the severity of inhalation injuries in adults. Burns. 2010;36:212-6.

14. Walker PF, Buehner MF, Wood LA, et al. Diagnosis and management of inhalation injury: an updated review. Critical Care. 2015;19:351.

15. Kagan RJ, Gamelli R, Saffle JR. DRG 504: the effect of 96 hours of mechanical ventilation on resource utilization. $J$ Burn Care Res. 2007;28(5):664-8.

16. Gravvanis Al, Tsoutsos DA, Iconomou TG, Papadopoulos SG. Percutaneous versus conventional tracheostomy in burned patients with inhalation injury. World J Surg. 2005;29:1571-1575.
17. Forster NA, Zingg M, Haile SR, Künzi W, Giovanoli P, Guggenheim M. 30 years later-does the ABSI need revision? Burns. 2011;37(6):958-63.

18. Tobiasen J, Hiebert JM, Edlich RF. The abbreviated burn severity index. Ann Emerg Med. 1982;11(5):260-2.

19. Waldmann V, Narayanan K, Combes N, Jost $D$, Jouven X, Marijon E. Electrical cardiac injuries: current concepts and management. Eur Heart J. 2018 Apr 21;39(16):1459-1465.

20. Pannucci CJ, Osborne NH, Jaber RM, Cederna PS, Wahl WL. Early fasciotomy in electrically injured patients as a marker for injury severity and deep venous thrombosis risk: an analysis of the National Burn Repository. J Burn Care Res. 2010; 31(6):882-887.

21. Parvathy G, Shaji CV, Kabeer KA, Prasanth SR. High-voltage electrocution causing bulbar dysfunction. J Neurosci Rural Pract. 2016;7(3):453-455.

22. Wesner ML, Hickie J. Long-term sequelae of electrical injury. Can Fam Physician. 2013;59(9):935-939.

23. Siddiqui AH, Farooq U, Siddiqui F. Curling Ulcer. [Updated 2021 Apr 17]. In: StatPearls [Internet]. Treasure Island (FL): StatPearls Publishing; 2021.

24. Church D, Elsayed S, Reid O, Winston B, Lindsay R. Burn wound infections. Clin Microbiol Rev. 2006;19(2):403-434. 